New Zealand, May 5, I869, and also in the "Annals and Mag. Nat. Hist." October I 870.

The skeleton of this animal has been lately placed among the fine series of Cetaceans in the Museum of the Royal College of Surgeons, thanks to the extremely liberal desire of Dr. Haast that it should be made as available as possible for scientific examination, comparison, and description, and to the generosity of Mr. Erasmus Wilson, F.R.S., a member of the Council of the College, in providing the means of adding it to the collection without expense to the Institution. A detailed and fully illustrated description of this skeleton formed part of the communication to the Zoological Society alluded to above, and will appear shortly in the "Transactions." All the characters of the skeleton agree generally with those of the other Ziphioids, but it appears in some respects to be a less specialised form, approaching somewhat nearer to the true dolphins, while Hyperoodon is at the other extremity of the series, being modified in the direction of the sperm whales. It has two teeth on each side of the lower jaw, situated near the front end or symphysis, which show nearly the same characteristic and peculiar structure as that described by Mr. Ray Lankester in the teeth of Mesoplodon Sowerbyi. The skull is far more symmetrical than in any other member of the group, and wants the great maxillary crests of Hyperoodon, and the dense ossification of the rostrum found in so many of the others. The cervical region is comparatively long, with the majority of its vertebræ free, the dorsals and ribs are ten in number, the lumber and caudals thirty-one, making forty-eight in all. Viewing the skeleton as a whole, the most striking feature is the small size of the head compared with the great length of the vertebral column, and the massiveness of the individual bones, especially of the lumbar and anterior caudal vertebræ. It presents in this respect a remarkable contrast to the sperm whale, which hangs near it in the museum, though agreeing generally with the other Ziphioids. As before mentioned, it is thirty feet in length, and, as Dr. Haast was able to observe, it agrees with its congeners in the nature of its food, for its stomach was found to contain about half a bushel of the horny beaks of cephalopods. The colour of the whole animal when fresh was of a deep velvety black, with the exception of the lower portion of the belly, which was greyish.

Extinct Ziphioids. - To the circumstance of the extreme density of the rostral portion of the skull of certain Ziphioids, owing to the firm ossification of the mesethmoid cartilage and its coalescence with the surrounding bones (the maxilla, premaxilla, and vomer) our knowledge of many of the ancient members of this group of whales is due. When all other portions of the skeleton have yielded to the destructive influence of time, these rostra, generally in the form of elongated and somewhat flattened cylinders, worn and eroded by the action of water, gravel, and sand, occasionally come to light to attest the presence of a former world of oceanic life. A few teeth also have been found which would appear to be referable to these same animals. The localities in which these occur in England are the Red Crag deposits of Suffolk. They are still more abundant, and in a much more perfect condition in the beds of corresponding age in the neighbourhood of Antwerp, which have fortunately been laid bare by the excavations made in the defensive works of that city. A magnificent series of these fossils containing many new forms has recently been added to the Brussels Museum, but until M. Ie Vicomte Du Bus, the accomplished late Director of the Museum, has completed the great task he has undertaken of determining and describing them, they are as little available for zoological science as if they still lay

In the bottom of the deep

Where fathom line could never touch the ground.

W. H. FLOWER
CONTINUITY OF THE FLUID AND GASEOUS STATES OF MATTER *

WHEN we find a substance capable of existing in two fluid states different in density and other properties, while the temperature and pressure are the same in both; and when we find also that an introduction or abstraction of heat without change of temperature or of pressure will effect the change from the one state to the other; and also find that the change either way is perfectly reversible, we speak of the one state as being an ordinary gaseous and the other as being an ordinary liquid sta ${ }^{4}$ of the same matter; and the ordinary transition from the one to the other we would designate by the terms boiling, or condensing; or occasionally by other terms nearly equivalent, such as evaporation, gasification, liquefaction from the gaseous state, \&c. Cases of gasification from liquids, or of condensation from gases, when any chemical alteration accompanies the abrupt change of density, are not among the subjects proposed to be brought under consideration in the present paper. In such cases I presume there would be no perfect reversibility in the process; and if so, this would of itself be a criterion sufficing to separate them from the proper cases of boiling or condensing at present intended to be considered. If now the fluid substance, in the rarer of the two states-that is, in what is commonly called the gaseous state-be still further rarefied, by increase of temperature or diminution of pressure, or be changed considerably in other ways by alterations of temperature and pressure jointly, without its receiving any abrupt collapse in volume, it will still, in ordinary language and ordinary mode of thought, be regarded as being in a gaseous state. Remarks of quite a corresponding kind may be made in describing various conditions of the fluid (as to temperature, pressure, and volume), which would in ordinary language be regarded as belonging to the liquid state.

Dr. Andrews (Phil. Trans. 1869) has shown that the ordinary gaseous and ordinary liquid states are only widely separated form: of the same condition of matter, and may be made to pass into one another by a course of continuous physical changes presenting nowhere any interruption or breach of continuity. If we denote geometrically all possible points of pressure and temperature jointly by points spread continuously in a plane surface, each point in the plane being referred to two axes of rectangular coordinates, so that one of its ordinates shall represent the tempe. rature, and the other the pressure denoted by that point; and if we mark all the successive boiling-or condensing-points of temperature and pressure as a continuous line on this plane; this line, which may be called the boiling line, will be a separating boundary between the regions of the plane corresponding to the ordinary liquid state and those corresponding to the ordinary gaseous state. But, by consideration of Dr. Andrews's experimental results, we may see that this separating boundary comes to an end at a point of pressure and temperature, which, in conformity with his language, may be called the critical point of pressure and temperature jointly ; and we may see that, from any ordinary liquid state to any ordinary gaseous state, the transition may be effected gradually by an infinite variety of courses passing round outside the extreme end of the boiling line.

Now it will be my cbief object in the present paper to state and support a view which has occurred to me, according to which it appears probable that, although there be a practical breach of continuity in crossing the line of boiling-points from liquid to gas or from gas to liquid, there may exist in the nature of things a theoretical continuity across this breach, having some real and true significance. This theoretical continuity, from the ordinary liquid state to the ordinary gaseous state, must be supposed to be such as to have its various courses passing through conditions of pressure, temperature, and volume in unstable equilibrium for any fluid matter theoretically conceived as homogeneously distributed while passing through the intermediate conditions. Such courses of transition, passing through unstable conditions, must be regarded as being impossible to be brought about throughout entire masses of fluids dealt with in any physical operations. Whether in an extremely thin lamina of gradual transition from a liquid to its own gas, in which it is to be noticed the substance would not be homogeneously distributed, conditions may exist in a stable state, having some kind of correspondence with the unstable conditions here theoretically conceived,

* "Considerations on the abrupt change at boiling or condensing in reference to the Continuity of the Fluid State of Matter," by Professor Jame. Thomson, LL.D., Queen's College, Belfast, read before the Royal Society, Nov. 16, 1871, 
will be a question suggested at the close of this paper in connection with some allied considerations.

It is first to be observed that the ordinary liquid state does not necessarily cease abruptly at the line of boiling-points, as it is well known that liquids may with due precautions be heated considerably beyond the boiling temperature for the pressure to which they are exposed. This condition is commonly manifested in the boiling of water in a glass vessel by a lamp placed below, when the temperature of the internal parts of the water, or, in other words, of the parts not exposed to contact with gaseous matter, rises considerably above the boiling-point for the pressure, and the water boils with bumping.* At this stage it becomes lesirable to refer to Dr. Andrews's diagram of curves, showing his principal results for carbonic acid, and to consider carefully some of the remarkable features presented by those curves. In doing so, we have first, in the case of the two curves for $13^{\circ} \cdot 1$ and $2 \mathrm{I}^{\circ}, 5$ which pass throngh the boiling interruption of continuity, to guard against being led by the gradually bending transition from the curve representing obviously the liquid state into the line seen rapidly ascending towards the curve repre- senting obviously the gaseous state, to suppose that this curved transition is in any way indicative of a gradual transition from the liquid towards the gaseous state. Dr. Andre ss has clearly pointed out, in describing those experimental curves, that the slight bend at about the commencement of the rapid ascent from the liquid state is to be ascribed to a trace of air unavoidably present in the carbonic acid; an $l$ that if the carbonic acid had been absolutely pure, the ascent from the liquid to the gaseous state would doubtless have been quite abrupt, and would have shown itself in his diagram by a vertical straight line, when we regard the co-ordinate axes for pressures and volumes as being horizontal and vertical respectively. Now in the diagram here submitted, the continuous curves (that is to say, those which are not dotted) are obtained from Dr. Andrews's diagram with the slight alteration of substituting, in accordance with the explanations just given, an abrupt meeting instead of the curved transition between the curve for the liquid state and the upright line which shows the boiling stage. Looking to either of the given curves which pass through boiling, and, for instance, selecting the curve for $13^{\circ} \cdot \mathrm{I}$, we perceive, from what has been said as to

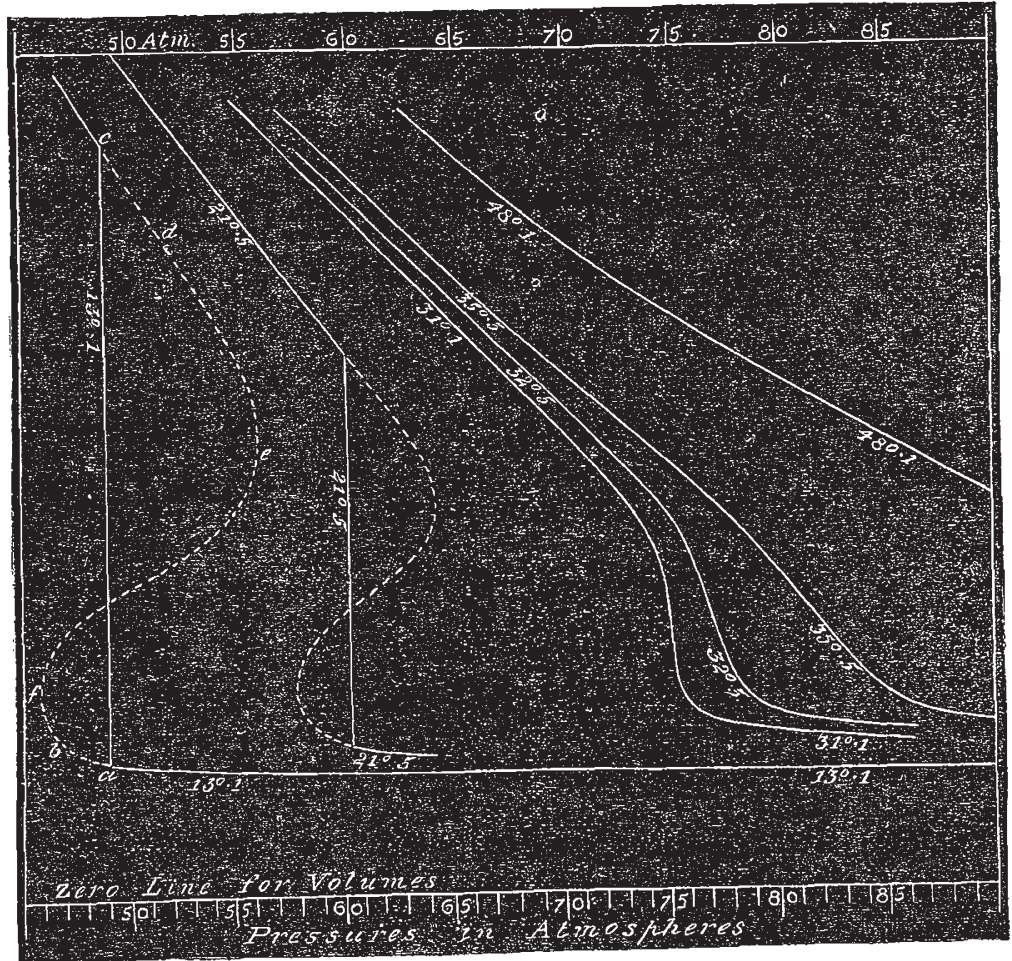

the conditions to which boiling by bumping is due, that for the the conditions to which bo this curve the lidate does not temperature pertaining to this curve the liquid state does not necessarily end at the boiling pressure for this temperature, and that thus in the diagram the curve showing volumes for the liquid state must not cease at the foot of the upright line which marks the boiling stage of pressure, but must extend continuch way for some distance at least, into lower pressures in $b$. But now the as is shown by the dotted continuation rom $a$ to question arises, Does this curve necessarily end at any particular question arises, Wnow that the extent of this curve in the direction point $b$ ? We know that the extent of this cur past $b$, along which the liquid volume will from $a$ towards or past continue to be represented before the explosive or bumstances, being to gas occurs, is very variable under different circumstances, being much affected by the presence of other fluids, even in small

* It has even been found by Dufour (Bibliothèque Universelle, Archives, * It has even been found by year $x 86 \mathrm{r}$, vol. . "Red in oil, so as neither to be in contact with any solid of water foating imm nor with any gaseous body, mase ordinary boiling-point, and occasionally various temperatures far abov $8^{\circ} \mathrm{C}$, without boiling. On this subject refer to so high a temperature as important researches of Donny, "Sur la ence may a'so be made to the impérence aux Corps solides," Ann. de Cohésion des Liquides et sur len A. 67, -July $28,187 \mathrm{I}$ quantities, as impurities in the fluid experimented on, and by the nature of the surface of the containing vessels, \&c.

The consideration of the subject may be facilitated, and aid towards the attainment of clear views of the mutual relations of temperature, presiure, and volume in a given mass of a fluid may be gained, by actually making, or conceiving there to be made, for carbonic acid, from the data supplied in Dr. Andrews' experimental results, a solid model consisting of a curved surface referred to three axes of rectangular co-ordinates, and formed so that the three co-ordinates of each point in the curved surface shall represent, for any given mass of carbonic acid, a temperature, a pressure, and a volume which can co-exist in that mass. It is to be noticed here that in his diagram of curves the results for each of the several temperatures experimented on are combined in the form of a plane-curved line referred to two axes of rectangular co-ordinates, one of each pair of co-ordinates representing a pressure, and the other representing the volume corresponding to that pressure at the temperature to which the curve belongs Now to form a model such as I am here recommend. ing, and have myself made, Dr. Andrews' curved lines are to be placed with their planes parallel to one another, and separated by intervals proportional to the differences of the temperatures to 
which the curves severally belong, and with the origins of coordinates of the curves situated in a straight line perpendicular to their planes, and with the axes of co-ordinates of all of them parallel in pairs to one another, and then the curved surface is to be formed so as to pass through those curved lines smoothly or evenly. "The curved surface so obtained exhibits in a very obvious way the remarkable phenomena of the voluminal conditions at and near the critical point of temperature and pressure, in comparison with the voluminal conditions throughout other parts of the range of gradually varying temperatures and pressures to which it extends, and even throughout a far wider range into which it can in imagination be conceived to be extended. It helps to afford a clear view of the nature and meaning of the continuity of the liquid and gaseous states of matter. It does so by its own obvious continuity throughout its expanse round the end of the range of points of pressure and temperature where an abrupt change of volume can occur by boiling or condensing. On the curved surface in the model Dr. Andrews' curves for the temperatures $13^{\circ} \cdot 1,21^{\circ} \cdot 5,31^{\circ} \cdot 1,32^{\circ} \cdot 5,35^{\circ} \cdot 5$ and $48^{\circ} \cdot 1$ Centigrade, which afford the data for its construction, may with advantage be all shown drawn in their proper places. The model admits of easily exhibiting in due relation to one another a second set of curves, in which each would be for a constant pressure, and in each of which the co-ordinates would represent temperatures and correspondiug voiumes. It may be used in various ways for affording quanitative relations interpolated among those more immediately given by the experiments.

We may now, aided by the conception of this model, return to the consideration of continuity or discontinuity in the curves in crossing the boiling stage. Let us suppose an indefinite number of curves, each for one constant ternperature, to be drawn on the model, the several temperatures differing in succession by very small intervals, and the curves consequently being sections of the curved surface by numerous planes closely spaced parallel to one another and to the plane containing the pair of co-ordinate axes for pressure and volume. Now we can see that, as we pass from curve to curve in approaching towards the critical point from the higher teruperatures, the tangent to the curve at the steepest point or point of inflection is rotating, so that its inclination to the plane of the co-ordinate axes for pressure and temperature, which we may regard as horizontal, increases till, at the critical point, it becomes a right angle. Then it appears very natural to suppose that in proceeding onwards past the critical point, to curves successively for lower and lower temperatures, the tangent at the point of inflection would continue its rotation, and the angle of its inclination, which before was acute, would now become obtuse. It seems much more natural to make such a supposition as this than to suppose that in passing the critical point from higher into lower temperatures the curved line, or the curved surface to which it belongs, should break itself asunder, and should come to have a part of its con. ceivable continuous course absolutely deficient. It thus seems natural to suppose that in some sense there is continuity in each of the successive curves by courses such as those drawn in the accumpanying diagram as dotted curves uniting continuously the curves for the ordinary gaseouss state with those for the ordinary liquid state.

The physical conditions corresponding to the extension of the curve from $a$ to some point $b$ we have seen are perfectly attainable in practice. Some extension of the gaseous curve into points of temperature and pressure below what I have called the boiling, or condensing line, as for instance some extension such as from $c$ to $d$ in the figure, I think we need not despair of practically realising in physical operations. As a likely mode in which to bring steam continuing gase tus to points of pressure and temperature at which it would collapse to liquid water if it had any particle of liquid water present along with it, or if other circumstances were present capable of affording some apparently requisite conditions for enabling it to make a beginning of the change of state, $\uparrow$ I would suggest the ad-

* For the practical execution of this, it is well to commence with a rectangular block of wood, and then carefitlly to pare it down, applying, from time to time, the various curves as templets to it; and proceedir g according to the general methods followed in a shipbuilder's modelling room in cutting out small models of ships according to curves laid down on paper as cross sections of the required model at various p!aces in its length

+ Tre principle thar "the particles of a substance, when existing all in on state onlv, and in continuous contact with one another, or in contact only under special circumstances with other substances, experience a difficulty of making a beginning of their change of state, whether from liquid to solid, or from liquid to gaseous, or probably also from solid to liquid, was proposed by me, and, so far as I am aware, was first announced in a paper by mitting speedily of dry steam nearly at its condensing tempera. ture for its pressure (or, to use a common expression, nearly saturcated) into a vessel with a piston or plunger, all kept hotter than the steam, and then allowing the steam to expand till by its expansion it would be cooled below its condensing point for its pressure; and yet I would suppose that if this were done with very careful precautions the steam might not condense, on account of the cooled steam being surrounded entirely with a thin film of superheated steam close to the superheated containing vessel. The fact of its not condensing might perhaps best be ascertained by observations on its volume and pressure. Such an experiment as that sketched out here would not be easily made, and unless it were conducted with very great precautions, there could be no reasonable expectation of success in its attempt; and perhaps it might not be possible so completely to avoid the presence of dust or other dense particles in the steam as to make it prove successful. I mention it, however, as appearing to be founded on correct principles, and as tending to suggest desirable courses for experimental researches. The overhanging part of the curve from $e$ to $f$ seems to represent a state in which there would be some kind of unstable equilibrium; and so, although the curve there appears to have some important theoretical significance, yet the states represented by its various points would be unattainable throughout any ordinary mass of the fluid. It seems to represent conditions of co-existent temperature, pressure, and volume, in which, if all parts of a mass of fluid were placed, it would be in equilibrium, but out of which it would be led to rush, partly into the rarer state of gas, and partly into the denser state of liquid, by the slightest inequality of temperature or of density in any part relatively to other parts. I might proceed to state, in support of these views, several considerations founded on the ordinary statical theory of capillary or superficial phenomena of liquids, which is dependent on the supposition of an attraction acting very intensely for very small distances, and causing intense pressure in liquids over and above the pressure applied by the containing vessel and measureable by any pressure-gauge. That statical theory has fitted remarkably well to many observed phenomena, and has sometimes even led to the forecasting of new results in advance of experiment. Hence, althongh dynamic or kinetic theories of the constitution and pressure of fluids now seem likely to supersede any statical theory, yet phenomena may still be discussed according to the principles of statical theory; and there may be considerable likelihood that conditions explained or rendered probable under the statical theory would have some corresponding explanation or confirmation under any true theory by which the statical might come to be superseded. With a view to brevity, how. ever, and to the avoidance of putting forward speculations perhaps part'y rash, though, I think, not devoid of real significance, I shall not at present enter on details of these considerations, but shall leave them with merely the slight suggestion now offered, and with the suggestion mentioned in an earlier part of the present paper, of the question whether in an extremely thin lamina of gradual transition from a liquid to its own gas, at their visible face of demarcation, conditions may not exist in a stable state having a correspondence with the unstable conditions here theoretically conceived.

\section{ALTERNATION OF GENERATIONS IN FUNGI}

$\mathrm{T}$ has long since been shown that certain fungi pass 1 through an alternation of generations on the same plant. The Rev. M. J. Berkeley demonstrated that in the case of the common "bunt" at least four consecutive forms of reproductive cellules were produced. In the majority of Uredines there are two forms of fruit, but these can scarcely be regarded as an alternation of generations, since there is no evidence that the spores of Trichobasis by germination, or otherwise, produce the bilocular spores of Puccinia. In Podisoma and Gymnosporangium (if the two genera are really distinct) the bilocular spores germinate freely and produce unilocular secondary spores. Prof.

me in the Proceedings of the Royal Society for November 24,3859 , and in a paper submitted to the British Association in the same year. In the present paper, at the place to which this note is annexed, I adduce the like further supposition that a diffculty of making a beginning of change of state from gaseous to liquid may also probably exist. 